\title{
Ectodermal-neural cortex 1 affects the biological function of lung cancer through the MAPK pathway
}

\author{
CHENGWEI WU*, XIANGHAI WANG ${ }^{*}$, XINGWEI WU and XINGWU CHEN \\ Department of Respiratory Medicine, Yijishan Hospital of Wannan Medical College, Wuhu, Anhui 241001, P.R. China
}

Received September 22, 2020; Accepted February 22, 2021

DOI: $10.3892 / \mathrm{ijmm} .2021 .4912$

\begin{abstract}
Ectodermal-neural cortex 1 (ENC1), a highly expressed protein in lung cancer tissues, was identified from the Cancer Genome Atlas (TCGA) database. The objective of the present study was to examine the effects of ENC1 on the biological functions of lung cancer cells. For this purpose, the expression of ENC1 was examined by RT-qPCR to compare mRNA expression levels between 28 lung cancer tissue samples and para-cancerous tissue samples. The association between ENC1 expression and clinicopathological features was evaluated between the 2 tissue types. Using RT-qPCR and western blot analysis, the expression of ENC1 was investigated in a normal lung cell line (16HBE) and 2 lung cancer cell lines (A549 and H1299). The effect of siRNA targeting ENC1 (si-ENC1) on the proliferation of A549 and H1299 cells was detected by CCK- 8 assay at the indicated time points. Transwell assay was used to measure the migration and invasion of A549 and H1299 cells following transfection with siRNA targeting ENC1 (si-ENC1). The expression levels of several proteins related to migration and invasion were examined by western blot analysis. A mouse model of subcutaneous tumor xenotransplantation was established in nude mice to examine the effects of ENC1 downregulation on cancer cells. The results revealed that the expression of ENC1 in lung cancer tissues and lung cancer cells was significantly higher than that in para-cancerous tissues and non-cancer lung cells, respectively. The knockdown of ENC1 in the A549 and H1299 cells using si-ENC1 significantly decreased cell proliferation, migration and invasion compared with the untransfected cells. The knockdown of ENC1 significantly downregulated the levels of matrix metalloproteinase (MMP)2, MMP9, $\mathrm{N}$-cadherin, p-c-Jun N-terminal kinase (JNK), p-extracellular signal-regulated kinase (ERK) and p-p38. The levels of
\end{abstract}

Correspondence to: Dr Xingwu Chen, Department of Respiratory Medicine, Yijishan Hospital of Wannan Medical College, 2 Zheshan W Road, Jinghu, Wuhu, Anhui 241001, P.R. China E-mail: cxw0028@126.com

*Contributed equally

Key words: lung cancer, extracellular signal-regulated kinase, c-Jun $\mathrm{N}$-terminal kinase, ectodermal-neural cortex 1
E-cadherin were upregulated. In the mouse lung tumor model, reduced levels of ENCl inhibited the growth of lung tumors. On the whole, the present study demonstrates that ENC1 is involved in the proliferation, migration and invasion of lung cancer cells, and may thus be an effective diagnostic target for certain cancers. The inhibition or reduction of ENC1 activity may represent a breakthrough in the treatment of lung cancer.

\section{Introduction}

Lung cancer is a malignant and fatal human cancer and is the leading cause of cancer deaths worldwide (1). Lung cancer is divided into 2 main types: Non-small cell lung cancer (NSCLC) and small cell lung cancer (SCLC). NSCLC is the most common type of lung cancer, accounting for approximately $85 \%$ of lung cancers, while the more malignant SCLC accounts for approximately $15 \%$ of cases (2). The majority of lung cancers are diagnosed at an advanced stage, which reduces the survival rate of patients $(3,4)$. Despite progress being made in the treatment of lung cancer, the long-term survival rates have not been improved significantly (5). A small fraction of patients with lung cancer undergo surgery at the initial stage; however, the majority of patients require chemotherapy as diagnosis when the disease is at an advanced stage. Survival rates are better when lung cancer is identified earlier during its progression.

Ectodermal-neural cortex 1 (ENC1) is an actin-binding protein expressed primarily in nerve cells (6). ENC1 is essential for adipocyte and neural crest cell differentiation $(6,7)$. Of note, $\mathrm{ENCl}$ reduces the aggregation and neurotoxicity of mutant Huntingtin proteins through the downregulation of p62 expression (8). Recently, ENC1 was shown to play a key role in malignancies. Studies have confirmed that $\mathrm{ENC1}$ is upregulated in various types of brain tumors, as well as in colon, ovarian and breast cancers (9-12). However, ENC1 has been shown to significantly increase the level of reactive oxygen species in ovarian cancer cells, inhibiting the proliferation, invasion and migration of these cells (11).

The mechanisms of action of ENC1 in the development of lung cancer remain unclear. In the present study, it was found that the ENC1 levels were increased in lung cancer. The downregulation of ENC1 significantly inhibited the proliferation and invasion of lung cancer cells, and altered the expression levels of a variety of other proteins. The silencing of ENC1 expression in A549 cells inhibited the growth of tumors when introduced into a mouse lung tumor 
model. These results are consistent with published evidence that ENC1 plays an important role in the regulation of the growth, proliferation and metastasis of cancers (9-12). ENC1 may be used as an effective marker for the presence of certain cancers and may be key to the discovery of effective treatments for cancer.

\section{Materials and methods}

Patients and sample collection. A total of 28 lung tumor tissues were obtained from patients with NSCLC at Yijishan Hospital (affiliated with Wannan Medical College) from April, 2018 to April, 2020 upon initial diagnosis of the cancer in each patient. None of the patients received any other specific treatment. Samples from tissues adjacent to the tumors were collected for use as controls. The present study was approved by the Ethics Committee of Yijishan Hospital. Informed consent was obtained from all patients.

Cells, cell culture and transfection. Lung cancer cell lines A549 (SCSP-503), H1299 (SCSP-589) and human bronchial epithelial cells 16HBE (FS-0400) were obtained from the cell bank of the Chinese Academy of Sciences (Shanghai, China). All cells were cultured in RPMI-1640 (Gibco; Thermo Fisher Scientific, Inc.) containing $10 \%$ fetal bovine serum and $100 \mu \mathrm{g} / \mathrm{ml}$ streptomycin (Gibco; Thermo Fisher Scientific, Inc.) at $37^{\circ} \mathrm{C}$ in a humidified atmosphere with $5 \% \mathrm{CO}_{2}$. For transfection, cells at approximately $60 \%$ confluency were transfected with $8 \mathrm{nmol} / 1$ siRNAs targeting ENC1 or ENC1 overexpression vector using the GenMute Transfection kit according to the manufacturer's instructions (SignaGen Laboratories) for $48 \mathrm{~h}$. Following 12-24 $\mathrm{h}$ of transfection, the culture medium was replaced with fresh medium for an additional $48 \mathrm{~h}$ of incubation at $37^{\circ} \mathrm{C}$ in a humidified atmosphere with $5 \% \mathrm{CO}_{2}$. siRNA against ENC1 (si-ENC1), non-specific siRNA (si-NC), ENC1 overexpression vector and vector were designed and synthesized by Guangzhou RiboBio Co., Ltd. The sequence of the siRNA (si-ENC1) was 5'-CTGCTACGATCCAACATTA-3'.

Histological analysis and immunohistochemistry (IHC). For hematoxylin and eosin (H\&E) staining (G1005, Servicebio), tissues were fixed by $10 \%$ neutral formalin for 1 day, dehydrated and embedded in paraffin, and then cut into 5- $\mu \mathrm{m}$-thick sections. After being dewaxed and treated with gradient alcohol, the sections were stained with hematoxylin for $5 \mathrm{~min}$ at room temperature and were washed with running water. Subsequently, alcohol solution with $1 \%$ hydrochloric acid and ammonia was employed. Subsequently, $1 \%$ eosin was utilized for further staining for $3 \mathrm{~min}$ at room temperature, gradient ethanol for section dehydration, and neutral balsam for section mounting, followed by microscope visualization (Nikon Eclipse E100; Nikon Corporation).

For IHC, the specimens were cut into sections (5- $\mu \mathrm{m}$-thick). All tissue samples were fixed with $10 \%$ neutral formalin and embedded in paraffin. Paraffin sections were dewaxed and the endogenous peroxides quenched with $0.3 \%$ hydrogen peroxide. They were then incubated with anti-ENC1 antibody (1:200, ab124902; Abcam) overnight (at $4^{\circ} \mathrm{C}$ ) and secondary antibody (1:200, 7074S; Cell Signaling Technology, Inc.) for $30 \mathrm{~min}$ (at $37^{\circ} \mathrm{C}$ ). The sections were stained with diaminobenzidine
(G1212; Servicebio). Images were collected under a microscope (Nikon Eclipse E100; Nikon Corporation).

Reverse transcription-quantitative PCR (RT-qPCR). Total RNA $(1 \mu \mathrm{g})$ was extracted from the cells and tissues using TRIzol reagent (Ambion; Thermo Fisher Scientific, Inc.) was reverse-transcribed into cDNA following the instructions provided with the cDNA synthesis kit (K1622; Fermentas; Thermo Fisher Scientific, Inc.). The concentration and quality of the RNA were measured usign a NanoDrop 2000 spectrophotometer (Thermo Fisher Scientific, Inc.), paired samples were adjusted to the similar concentration for use. The mRNA level of GAPDH was used as an internal control. Relative mRNA expression levels calculated normalized to GAPDH. All experiments were performed in triplicate. qPCR was performed at $95^{\circ} \mathrm{C}$ for $10 \mathrm{~min}$ followed by 40 cycles at $95^{\circ} \mathrm{C}$ for $15 \mathrm{sec}$ and at $60^{\circ} \mathrm{C}$ for approximately $1 \mathrm{~min}$ by using the QuantiNova $^{\mathrm{TM}}$ SYBR $^{\circledR}$-Green PCR kit according to the manufacturer's instructions (Qiagen $\mathrm{GmbH}$ ). The ENC1 primers were as follows: Forward, 5'-TGGTTGGAGGATACTTTG GCATTCAG-3' and reverse, 5'-TAGGAATCAGCGAGTACG GGACAG-3'. The GAPDH primers were as follows: Forward, 5'-CTGGGCTACACTGAGCACC-3' and reverse, 5'-AAG TGGTCGTTGAGGGCAATG-3'. Relative mRNA expression was calculated using the $2^{-\Delta \Delta C q}$ method, as previously described (13). GAPDH served as an internal control.

Western blot analysis. Whole cells (A549 and H1299) and tissues (lung cancer and para-cancerous tissues) were lysed using cell lysis buffer (KeyGen Biotech. Co. Ltd.). The protein concentration was measured using a BCA protein assay kit (Beyotime Institute of Biotechnology). Protein samples (30 $\mu \mathrm{g})$ were separated by $10 \%$ SDS-PAGE and proteins transferred to PVDF membranes (Bio-Rad Laboratories, Inc.). Membranes were blocked with 5\% non-fat milk (Bio-Rad Laboratories, Inc.) for $1 \mathrm{~h}$ at room temperature, and subsequently incubated with diluted primary antibody at $4^{\circ} \mathrm{C}$ overnight. The membranes were incubated with HRP-conjugated secondary antibodies (1:2,000, 7074S, Cell Signaling Technology, Inc.) for $1 \mathrm{~h}$ at room temperature. Primary antibodies used in the present study included the following: ENC1 was obtained from Abcam (1:1,000, ab124902); N-Cadherin (1:1,000, 13116S), E-Cadherin $(1: 1,000,3195 S)$, matrix metalloproteinase (MMP)2 (1:1,000, 40994S), MMP9 (1:1,000, 13667S), p-extracellular signal-regulated kinase (ERK) $(1: 1,000$, AP0485), ERK (1:1,000, 4695T), p-c-Jun N-terminal kinase (JNK) $(1: 1,000,4668 \mathrm{~T})$, JNK $(1: 1,000,9252 \mathrm{~T}), \mathrm{p}-\mathrm{p} 38$ (1:1,000, $4511 \mathrm{~T}), \mathrm{p}-38(1: 1,000,8690 \mathrm{~T})$ and $\beta$-actin $(1: 1,000,3700 \mathrm{~T})$ were obtained from Cell Signaling Technology, Inc. The blots were visualized using an ECL kit (Bio-Rad Laboratories, Inc.). Bands were visualized by an enhanced chemiluminescence detection system (Bio-Rad Laboratories, Inc.) and quantified using ImageJ software (NIH) and normalized to the internal control, $\beta$-actin.

Cell viability and proliferation assay. The viability of lung cancer cell lines (A549 and H1299) was determined by CCK-8 assay (Bioss). Following transfection with the siRNAs for 24, 48 and $72 \mathrm{~h}, 10 \mu \mathrm{l} \mathrm{CCK}-8$ were added to each well followed by incubation for $3 \mathrm{~h}$ at $37^{\circ} \mathrm{C}$ according to manufacturer's 
instructions. The absorbance at $450 \mathrm{~nm}$ was determined using a multi-detection microplate reader (Bio-Tek Instruments, Inc.). The proliferation of lung cancer cell lines (A549 and H1299) was evaluated using the EdU kit (C10310-1/-2/-3; Guangzhou RiboBio Co., Ltd.). The transfected lung cancer cell lines (A549 and H1299) were seeded in 96-well plates and incubated for $24 \mathrm{~h}$ at $37^{\circ} \mathrm{C}$ in a humidified atmosphere with $5 \% \mathrm{CO}_{2}$. The cell culture medium was then supplemented with $100 \mu 150 \mu \mathrm{M}$ EdU and cells were incubated for a further $2 \mathrm{~h}$ at $37^{\circ} \mathrm{C}$ in a humidified atmosphere with $5 \% \mathrm{CO}_{2}$. The cells were then fixed with $4 \%$ paraformaldehyde at room temperature for $30 \mathrm{~min}$, followed by $0.5 \%$ Triton X-100 to permeabilize cell. Nucleic acids were stained with DAPI dye at room temperature for $5 \mathrm{~min}$. Signals were detected using an inverted fluorescence microscope (Olympus IX83, Olympus Corporation). Following transfection with the siRNAs for $48 \mathrm{~h}$, the proportion of proliferating cells in each group was determined. The intensity was determined using Image J software (NIH).

Cell invasion and migration assay. Transwell membrane filters ( $8 \mu \mathrm{m}$ pore size, EMD Millipore) were used for the invasion and migration assays. For cell invasion assay, $50 \mu 1$ Matrigel (BD Biosciences) were added to the upper chambers and incubated at $37^{\circ} \mathrm{C}$ for $1 \mathrm{~h}$, while Matrigel was not added for cell migration assay. Approximately $5 \times 10^{4}$ si-ENC1-transfected A549 and H1299 cells in a volume of $100 \mu \mathrm{l}$ were seeded in the upper chamber without serum. The Transwell chambers were then placed in 24-well plates and $600 \mu \mathrm{l}$ RPIM-1640 medium with $10 \%$ FBS were added to the lower chambers. Following incubation for $24 \mathrm{~h}$ at $37^{\circ} \mathrm{C}$ in a humidified atmosphere with $5 \% \mathrm{CO}_{2}$, the non-migrated cells were removed using a cotton swab. Migrated cells were fixed with $4 \%$ PFA for $30 \mathrm{~min}$, then stained with crystal violet solution for $20 \mathrm{~min}$. After washing with phosphate-buffered saline, 5 random fields of cells were imaged and quantified under a CNikon Eclipse E100; Nikon Corporation). The average of the cells from 5 fields was used for statistical analysis.

Tumor xenotransplantation experiment. Subcutaneous xenografts were created in the ventral region of female BALB/C nude mice (weighting approximately 20-22 g each) at 4 weeks of age. The mice were allowed to adapt in the SPF environment (temperature: $23-24^{\circ} \mathrm{C}$, humidity: $30-50 \%$ ), and food and water were freely available throughout the study. BALB/C nude mice were divided randomly into 2 groups ( $n=5$ per group). A total of $5 \times 10^{6}$ A549 cells with stable expression of sh-NC or sh-ENC1 were implanted. Tumor nodules were monitored once a week and tumor volumes were estimated using the following formula: Volume=longest diameter $\mathrm{x}$ (smallest diameter $)^{2} / 2$.

The mice were sacrificed after 6 weeks and the tumors were collected. All animals were euthanized by an intravenous injection of $100 \mathrm{mg} / \mathrm{kg}$ pentobarbital sodium. The weight of the tumors was recorded and the tumors fixed in 4\% PFA overnight, dehydrated and embedded in paraffin. The samples were sectioned and stained with H\&E as described above. The animal research was approved by the Ethics Review Committee of Wannan Medical College.

TCGA validation of ENC1. The expression level of ENC1 was verified using the UALCAN lung cancer database (http://ualcan.path.uab.edu/index.html). The TCGA survival data and Kaplan plot for ENC1 were verified using the OncoLnc lung cancer database (http://www.oncolnc.org/).

Microarray data. The microarray data have been deposited on the GEO database (GEO-GSE165972).

RNA sequencing. Total RNA was extracted using TRIzol reagent (Invitrogen; Thermo Fisher Scientific, Inc.) according to the manufacturer's instructions. The RNA concentration was determined using a Bioanalyzer 2100 and RNA 1000 Nano LabChip kit (Agilent Technologies, Inc.) with RIN number $>$ 7.0. RNA sequencing and analysis were provided by LC-bio. Bioinformatics analyses (GO and KEGG analyses) were performed using the OmicStudio tools at https://www. omicstudio.cn/tool.

Statistical analysis. Statistical analyses were performed using GraphPad Prism 5.0. All values represent at least 3 independent experiments and are presented as the means \pm SD. Differences between means were analyzed using a Student's t-test. P-values $<0.05$ were considered to indicate statistically significantly differences.

\section{Results}

High expression of ENC1 in lung cancer tissues and cells. The mRNA expression levels of ENC1 between the lung cancer and para-cancerous tissues were compared using the TCGA database. As shown in Fig. 1A and B, the mRNA levels of $\mathrm{ENC1}$ in the cancer tissues were increased compared to those in the para-cancerous tissues. Analysis using the TCGA database also revealed that the lung cancer samples with a higher ENC1 expression (red bar) exhibited a worse overall survival, but the difference was not significant (Fig. 1C). Subsequently, the mRNA expression of ENC1 was compared between cancer and para-cancerous tissues obtained from lung cancer patients. The expression of ENC1 was determined by RT-qPCR and IHC (Fig. 1D-F). As shown in Fig. 1D and E, the expression of ENC1 was significantly increased in lung tumor tissues compared to para-cancerous tissues, and the expression of ENC1 was significantly increased in stage III cancer tissues compared to stage II cancer tissues. The association between the clinicopathological features of patients with NSCLC and the expression levels of ENC1 was also investigated (Table I), and the expression of ENC1 was only related to the M phase, not to sex, age, tumor size or $\mathrm{N}$ stage. In addition, the expression of ENC1 in a normal human bronchial epithelium cell line (16HBE), and lung cancer cell lines (A549 and H1299) was examined by RT-qPCR and western blot analysis (Fig. 1G and $\mathrm{H}$ ). The results revealed that the expression of ENC1 in the A549 and H1299 cells was markedly higher than that in the $16 \mathrm{HBE}$ cells. These results suggest that ENC1 is highly expressed in lung cancer cells and tissues.

Gene expression profiling of si-ENC1-transfected A549 cells. Overall gene expression was evaluated in siRNA-transfected A549 cells. The transcriptome of the A549 cells transfected with si-NC and si-ENC1 was compared. In total, there were 1,404 differentially expressed genes (DEGs) between the cells 
Table I. Associations between ENC1 expression and the clinicopathological parameters of patients with lung adenocarcinoma.

\begin{tabular}{|c|c|c|c|}
\hline Characteristic & No. of patients & ENC1 expression $\left(2^{-\Delta \Delta C q}\right)$, means \pm SD & P-value \\
\hline $\operatorname{Sex}$ & & & 0.42 \\
\hline Male & 17 & $4.828 \pm 3.672$ & \\
\hline Female & 11 & $3.530 \pm 4.688$ & \\
\hline Age, years & & & 0.262 \\
\hline$\leq 65$ & 18 & $3.641 \pm 4.080$ & \\
\hline$>65$ & 10 & $5.462 \pm 4.035$ & \\
\hline Tumor size $(\mathrm{cm})$ & & & 0.728 \\
\hline$\leq 3$ & 7 & $3.809 \pm 2.644$ & \\
\hline$>3$ & 21 & $4.461 \pm 4.507$ & \\
\hline $\mathrm{N}$ stage & & & 0.794 \\
\hline NO & 13 & $4.374 \pm 2.920$ & \\
\hline $\mathrm{N} 1-\mathrm{N} 3$ & 15 & $4.841 \pm 5.409$ & \\
\hline M stage & & & 0.037 \\
\hline II & 20 & $3.094 \pm 2.405$ & \\
\hline III & 8 & $6.067 \pm 4.399$ & \\
\hline
\end{tabular}

transfected with si-NC and si-ENC1 (Fig. 2A). Hierarchical clustering of the DEGs separated the 2 groups (Fig. 2B). GO and KEGG analyses revealed changes in gene expression related to cancer development, including cell adhesion, cell communication, cell transduction, apoptosis and small cell lung cancer (Fig. 2C-F). These results suggested that downregulated ENC1 could affect the progression of lung cancer.

Downregulation of ENC1 inhibits the migration and invasion of NSCLC cells. To examine the effects of si-ENC1 on the invasion and migration of A549 and H1299 cells, Transwell assay was performed. Following transfection with si-ENC1, the mRNA and protein levels of ENC1 were determined in the A549 and H1299 cells. As shown in Fig. 3A and B, the mRNA levels of ENC1, as well as the protein levels were significantly reduced by transfection with si-ENC1. The results of Transwell assay revealed that the downregulation of ENC1 significantly inhibited the migratory and invasive ability of the A549 and H1299 cells compared to the control group (Fig. 3C-J). MMP2 and MMP9 are the primary enzymes that degrade type IV collagen, playing an important role in the vascularization, invasion, and metastases of tumor cells $(14,15)$. They have been shown to be closely related to the development of lung cancer (16). In the present study, following transfection with si-ENC1, the MMP2 and MMP9 protein levels were determined in the A549 and H1299 cells. As shown in Fig. 3K-M, the protein levels of MMP2 and MMP9 were decreased following transfection with si-ENC1. Epithelial-mesenchymal transition (EMT) plays an important role in the process of tumor cell metastasis, which has been widely recognized. The decrease in E-cadherin expression and the increase in $\mathrm{N}$-cadherin expression are considered as key factors of EMT. In the present study, as shown in Fig. 3K-M, following transfection with si-ENC1, the expression of $\mathrm{N}$-cadherin was decreased, while that of E-cadherin was increased.
Downregulation of ENC1 inhibits the proliferation of NSCLC cells. The proliferation of the A549 and H1299 cells following transfection with si-ENC1 was assessed. The results of CCK- 8 assay revealed that the downregulated expression of ENC1 inhibited the proliferation of the A549 and H1299 cells (Fig. 4A and B). Similar results were also obtained using EdU detection (Fig. 4C-F). These results suggest that ENC1 plays a significant role in the proliferation of NSCLC cells.

Downregulation of ENC1 affects the MAPK pathway. Subsequently, the present study investigated whether si-ENC1 can affect the regulation of the MAPK pathway. As shown in Fig. 5, transfection of the A459 and H1299 cells with si-ENC1 significantly inhibited the phosphorylation of JNK, ERK and p38. However, there was no significant change in the total protein levels of JNK, ERK and p38. These results suggest that ENC1 inhibits the migration, invasion and proliferation of NSCLC cells by affecting the pathway downstream of MAPK.

Downregulation of ENC1 inhibits the growth of NSCLC in a mouse model. To determine the effect of $\mathrm{ENC1}$ on the growth of tumors in vivo, a subcutaneous xenograft tumor model was established. The A549 cells, which were transfected with sh-ENC1 or sh-ctrl, were introduced into nude mice. As shown in Fig. 6A and B, the growth of the tumors in the sh-ENC1 group was significantly inhibited and the weight of the tumors was significantly lower than that in the sh-ctrl group (Fig. 6C). In addition, tumor tissue structures are shown in Fig. 6D. Subcutaneous transplantation of tumor in nude mice was successful. The cancer cells were diffusely distributed and formed adenoid structures, with obvious atypia, pathological mitosis and focal necrosis. The expression of E-cadherin increased and that of $\mathrm{N}$-cadherin decreased in the sh-ENC1 group compared with the sh-ctrl group (Fig. 6E and F). In 


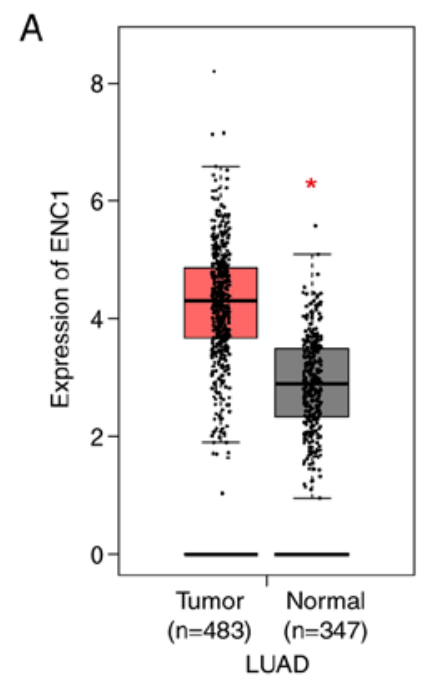

B

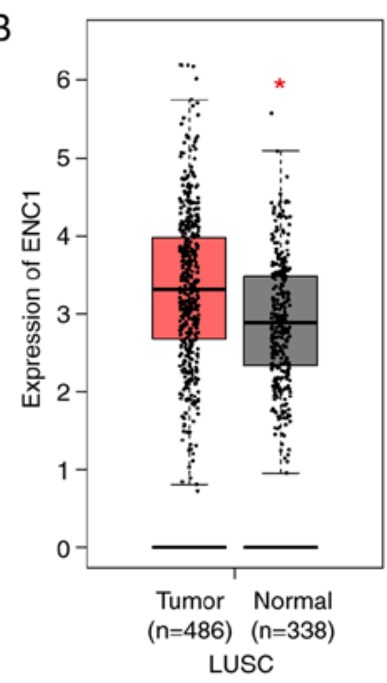

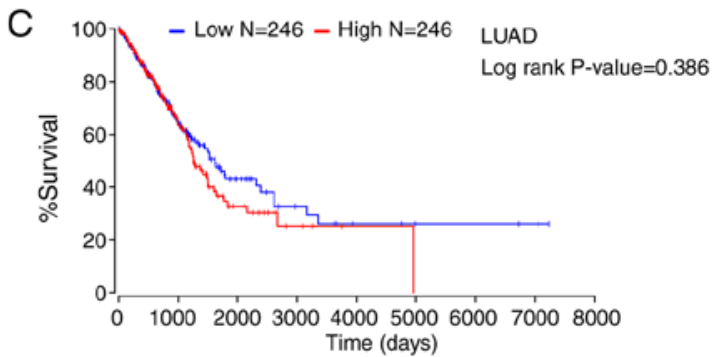

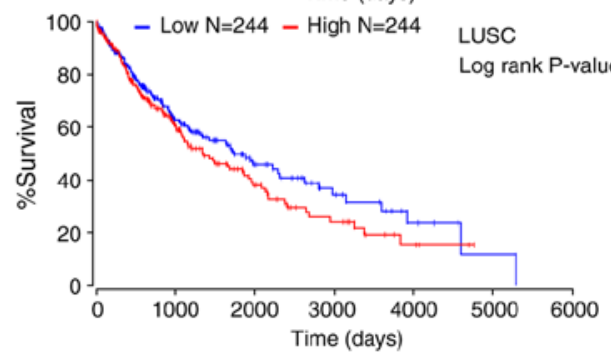

$\mathrm{D}$

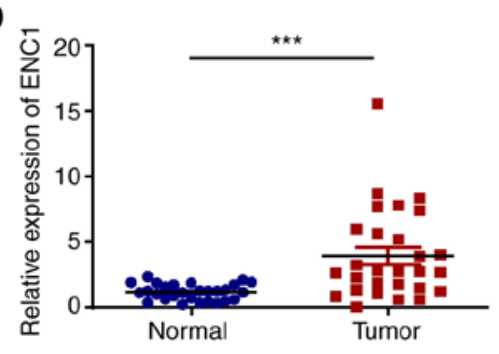

$\mathrm{F}$

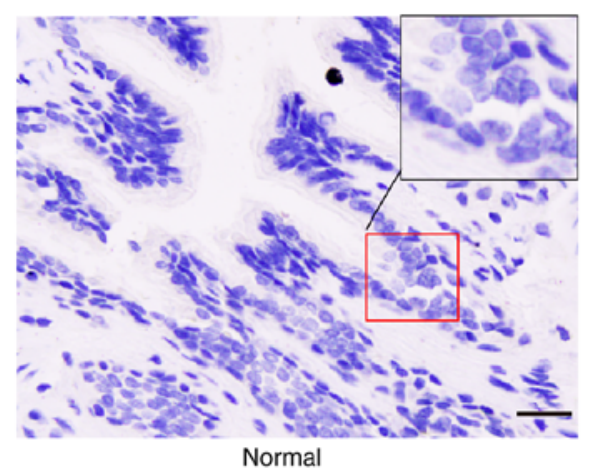

E
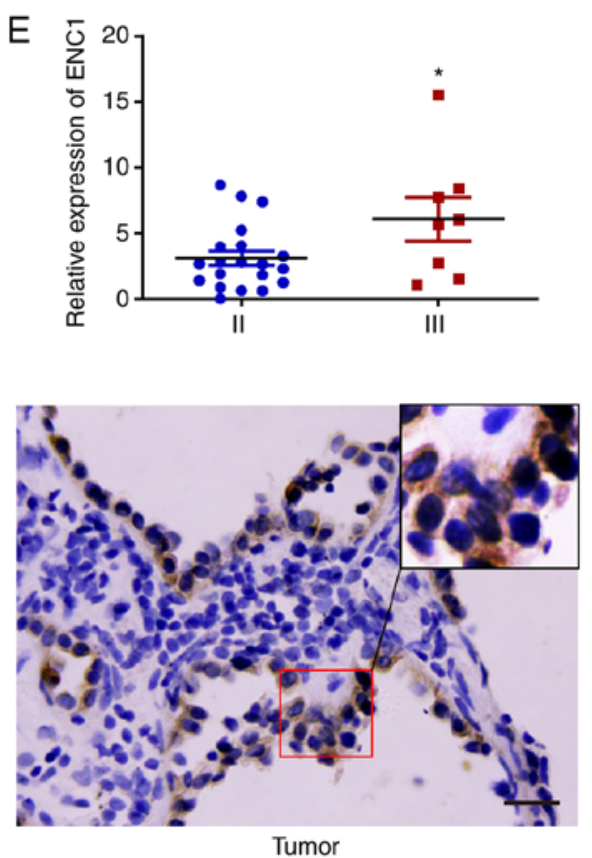

G

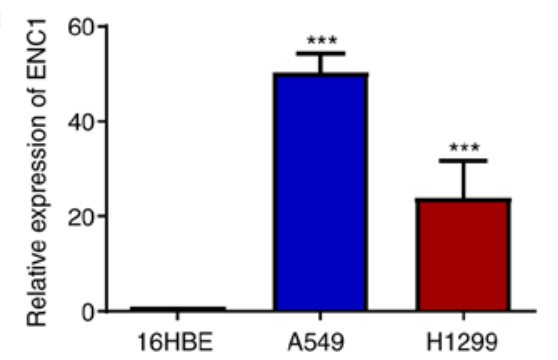

$\mathrm{H}$

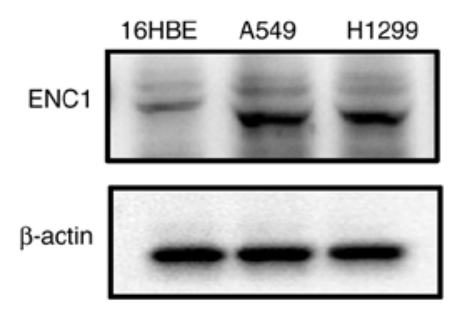

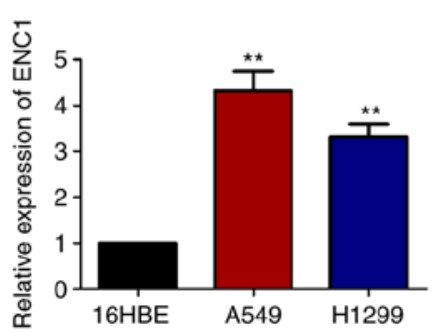

Figure 1. High expression of ENC1 in lung cancer tissues and cells. (A and B) The expression of ENC1 between lung cancer and para-cancerous tissue from the TCGA database. (C) Kaplan-Meier survival plot of overall survival of lung cancer in TCGA database, categorized according to ENC1 gene expression (high vs. low, based on mean expression). (D) The expression of ENC1 in normal and tumor tissues by RT-qPCR. (E) The expression of ENC1 in tumor tissues between stage II and III by RT-qPCR. (F) The expression of ENC1 in normal and tumor tissues by immunohistochemical analysis. (G and H) The expression of ENC1 between normal cell line and lung cancer cell lines by RT-qPCR and western blot analysis. Scale bar, $50 \mu \mathrm{m}$; ${ }^{*} \mathrm{P}<0.05,{ }^{* * * *} \mathrm{P}<0.001$ vs. normal group; ${ }^{* *} \mathrm{P}<0.01$ vs. 16HBE cell group; ENC1, ectodermal-neural cortex 1.

addition, the expression of both MMP2 and MMP9 was decreased in the sh-ENC1 group (Fig. 6G and H). These experiments suggest that ENC1 is a key gene mediating the growth and metastasis of lung cancer.
Upregulation of ENCl enhances the proliferation, migration and invasion of NSCLC cells. The effects of a high expression of ENC1 on NSCLC cells were then investigated. An ENC1-overexpression plasmid (OE-ENC1 group) and an NC 

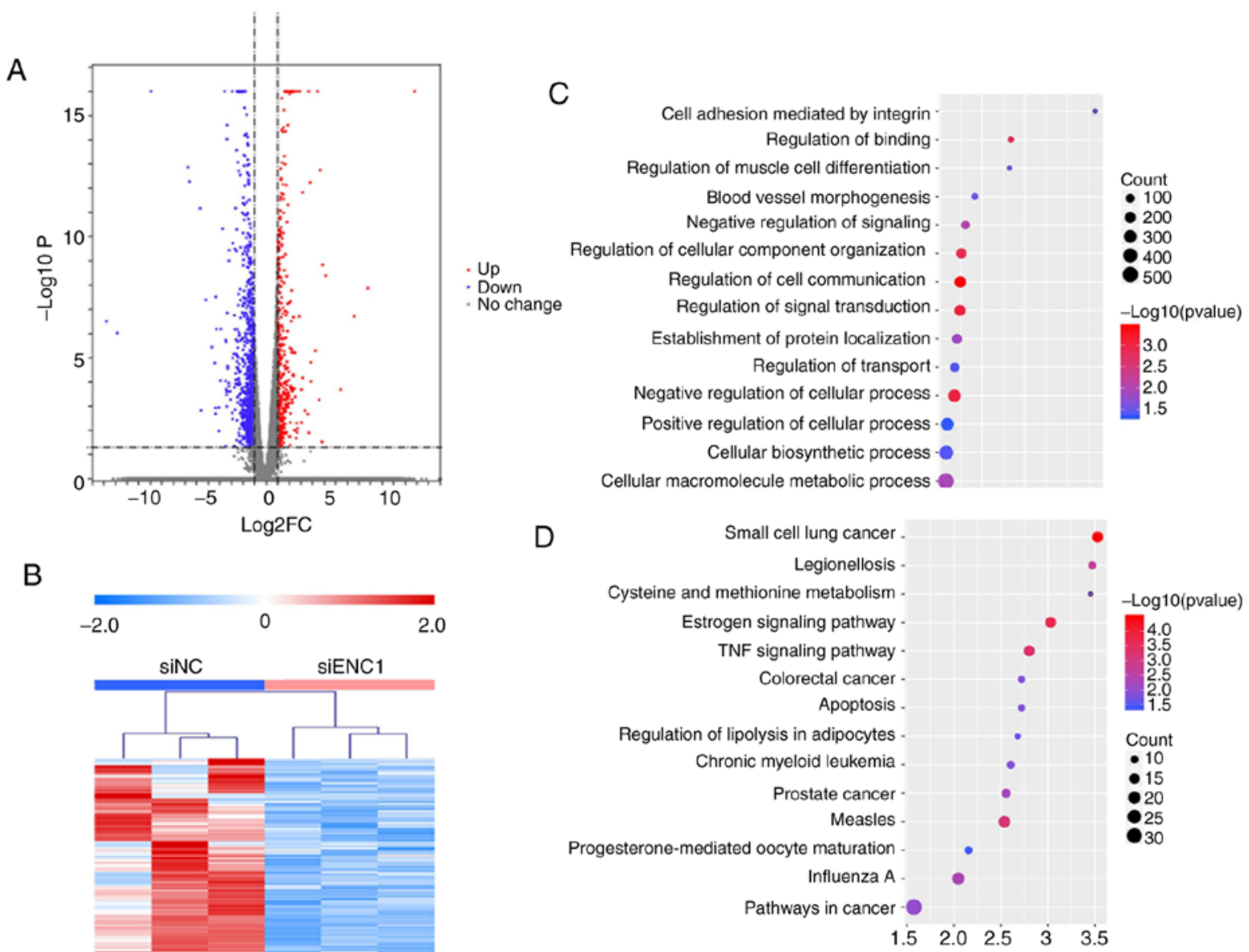

B

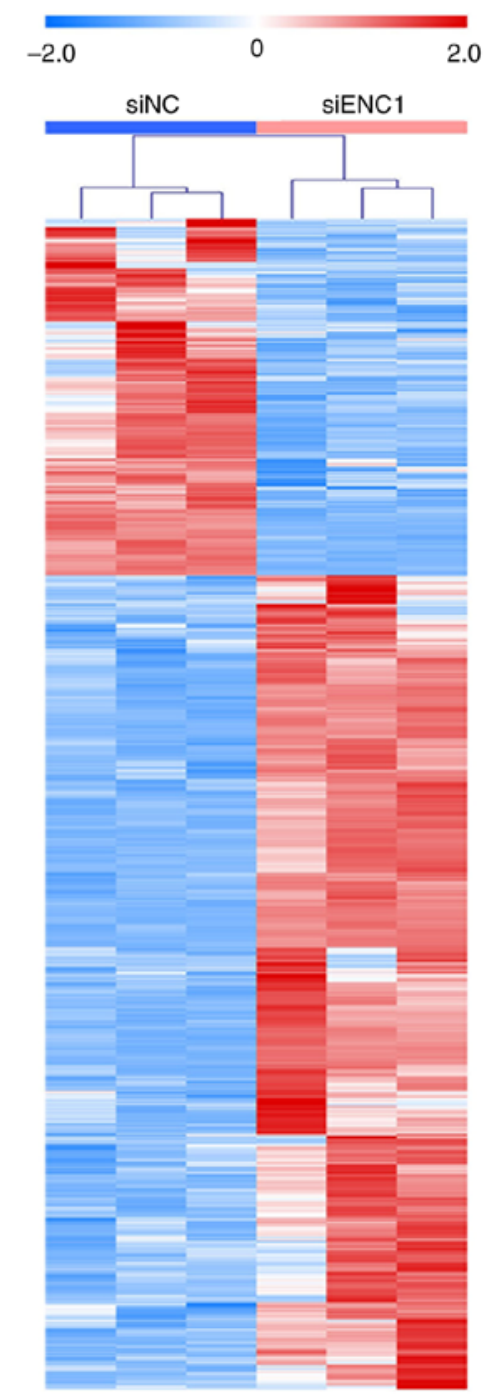

$\mathrm{E}$
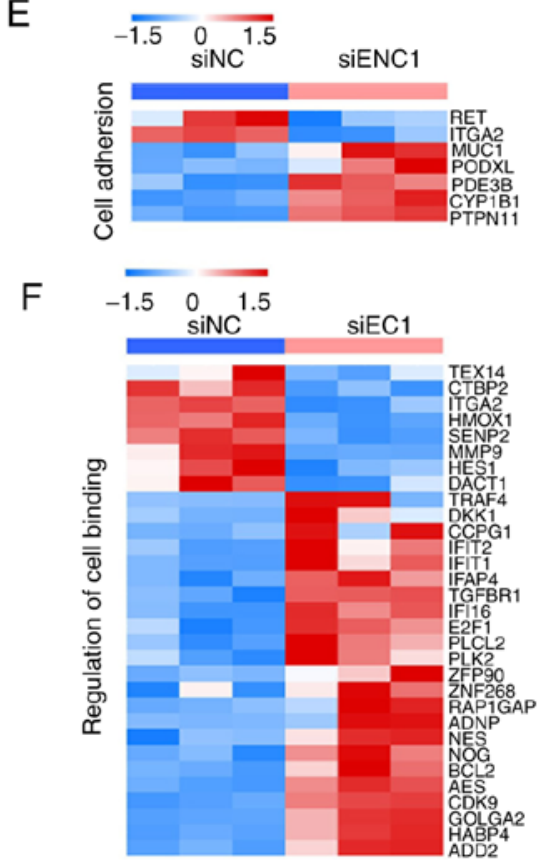

Figure 2. Gene expression profiling of si-ENC1-transfected A549. (A) Volcano plot of all genes detected in transcriptome analysis. (B) Heatmap represents the magnitude of gene expression of 1404 genes $(\mathrm{P}<0.05, \operatorname{LogFC} \geq 1$ o $\mathrm{r} \leq-1)$. (C and $\mathrm{D})$ Relative fold change of genes representing key pathways identified by $\mathrm{GO}$ and KEGG analyses. (E and F) Heatmap represents magnitude of gene expression of cell adhesion and regulation of cell binding. ENC1, ectodermal-neural cortex 1.

plasmid (OE-NC group) were transfected into the A549 and H1299 cells. The ENC1 mRNA and protein levels were significantly increased in both cell lines following transfection with OE-ENC1 (Fig. 7A and B). The increased expression of ENC1 induced the proliferation of A549 and H1299 cells, as shown by CCK-8 assay (Fig. 7C). Similar results were obtained using the EdU detection kit (Fig. 7D). As shown in Fig. 7E and F, the upregulation of ENC1 significantly enhanced the migration 

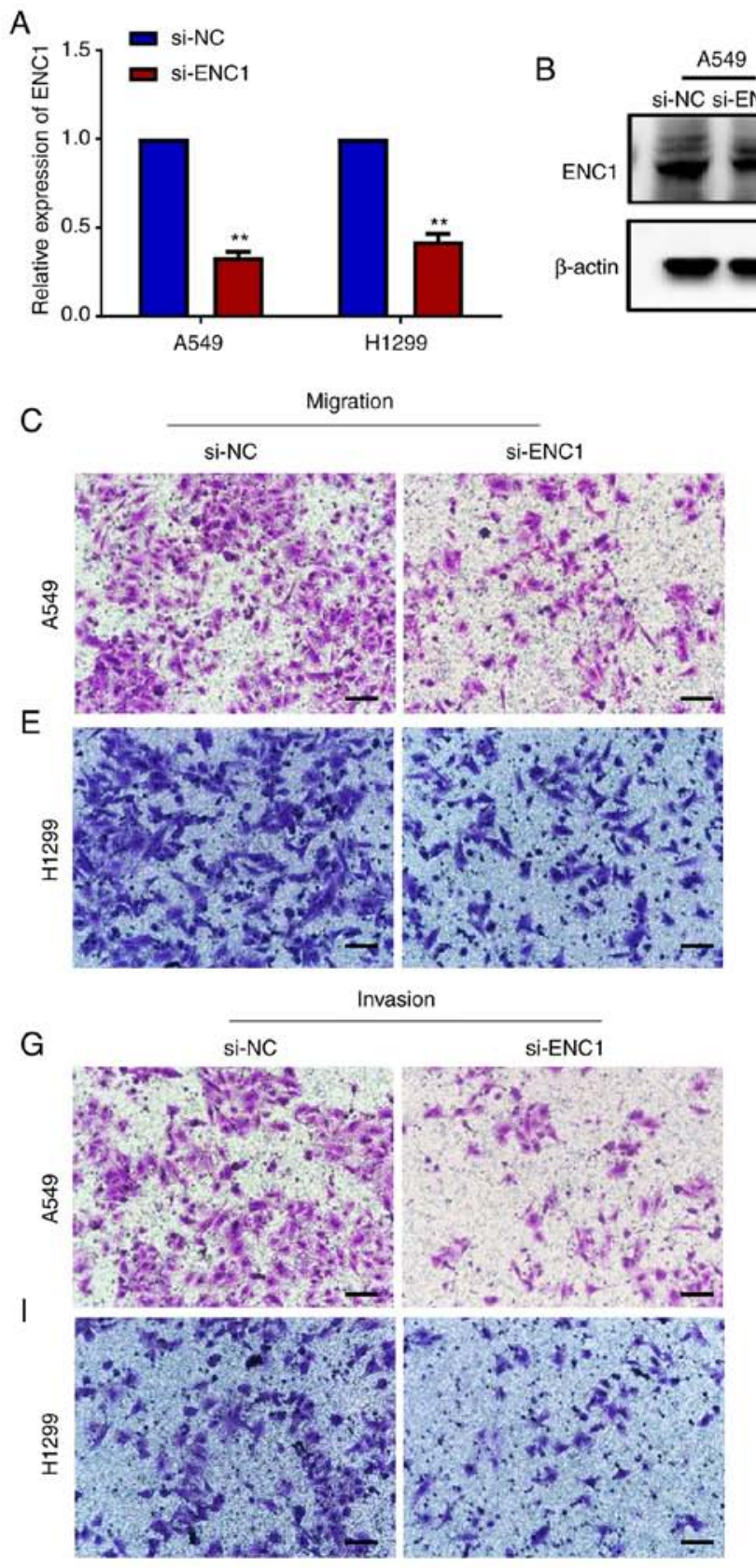

D

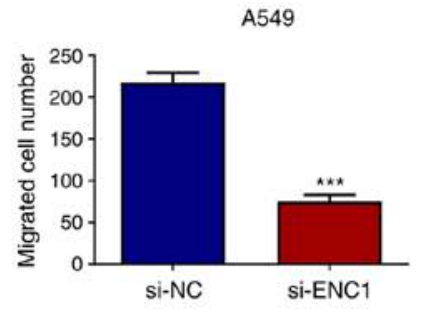

$\mathrm{F}$

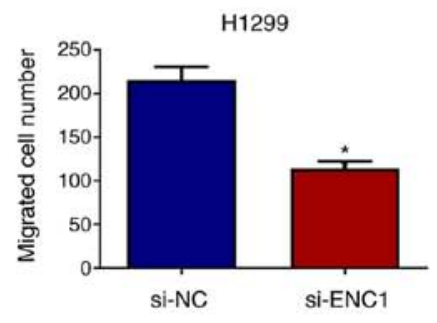

$\mathrm{H}$
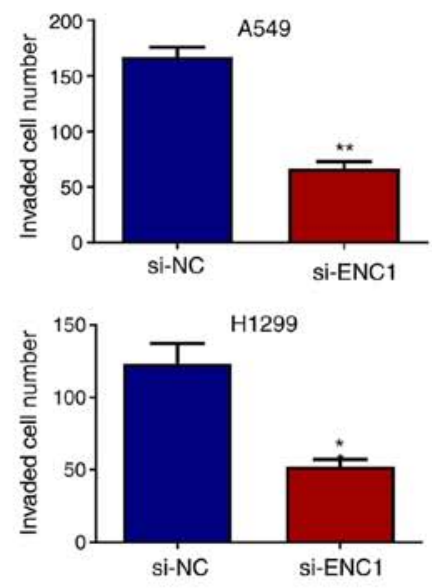

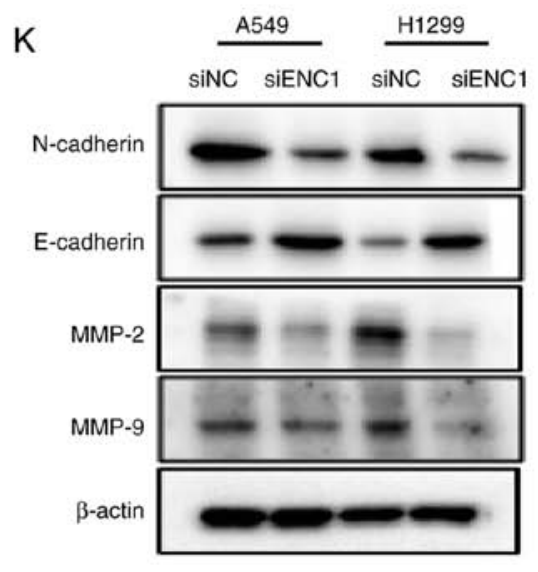

L

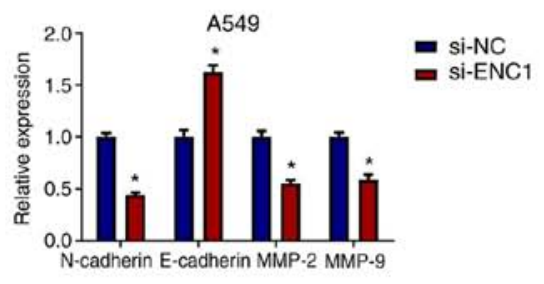

M

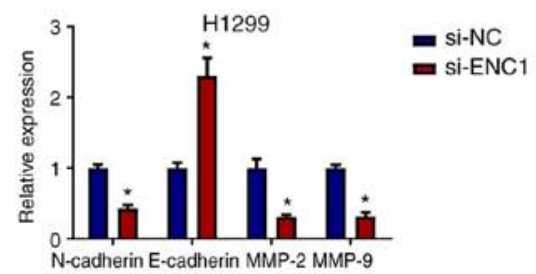

Figure 3. Downregulation of ENC1 inhibits the migration and invasion of NSCLC cells. (A) Knockdown efficiency of ENC1 was quantified in A549 and H1299 by RT-qPCR. (B) Knockdown efficiency of ENC1 was quantified in A549 and H1299 by western blot analysis. (C-J) The effects of ENC1 deficiency on cell migration and invasion were investigated by Transwell assay. (K-M) The effects of ENC1 deficiency on the expression of N-cadherin, E-cadherin, MMP2 and MMP9 were investigated by western blot analysis. ${ }^{\mathrm{P}}<0.05,{ }^{* *} \mathrm{P}<0.01,{ }^{* * * *} \mathrm{P}<0.001$ vs. si-NC group. ENC1, ectodermal-neural cortex 1; NSCLC, non-small cell lung cancer. 
A

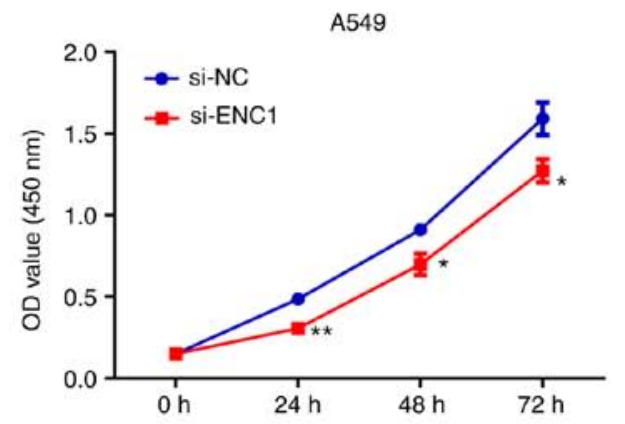

B

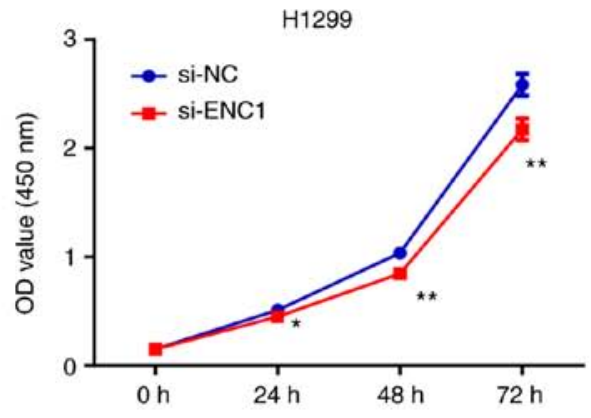

C

C
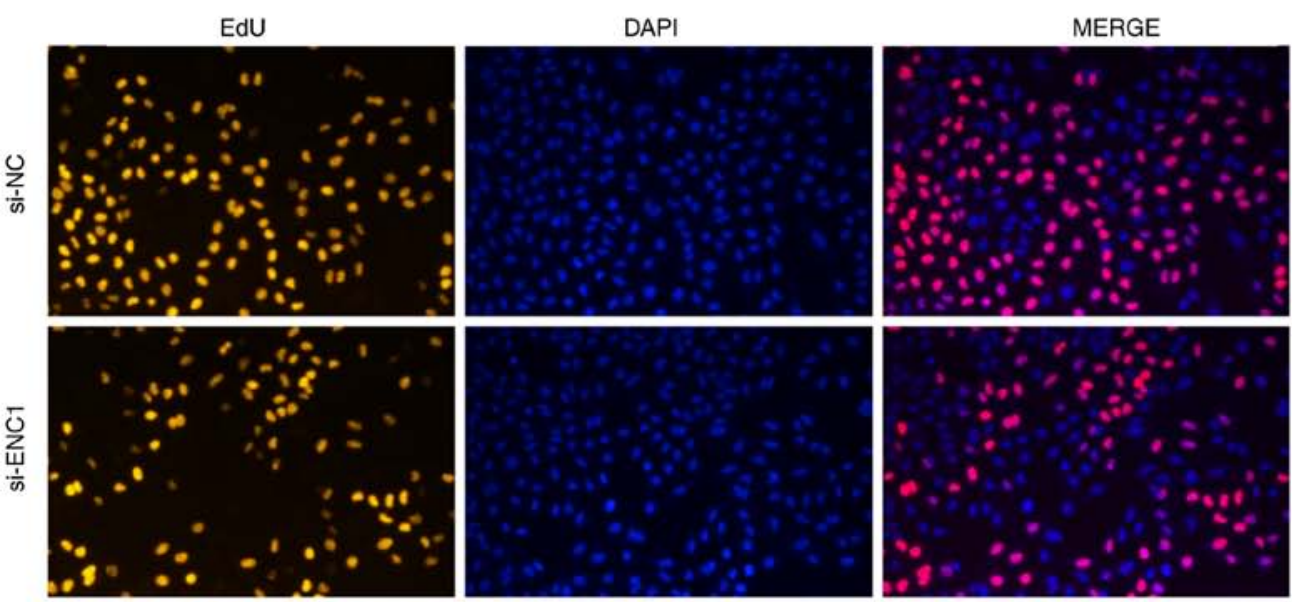

爱

D
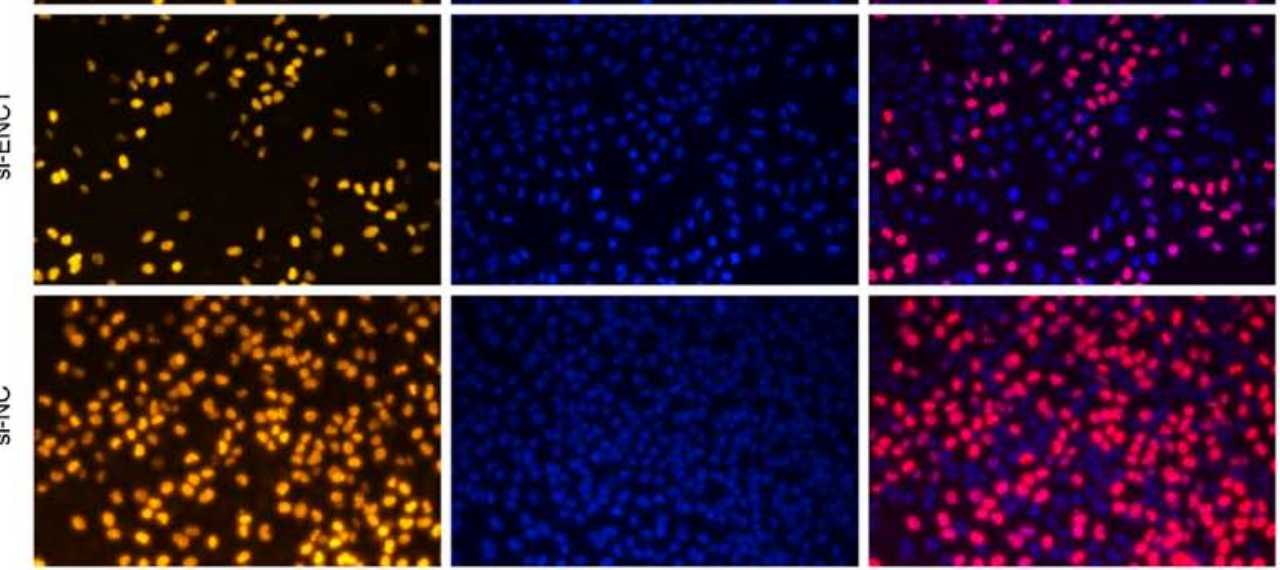

$\stackrel{\text { 오 }}{\frac{\text { I }}{I}}$
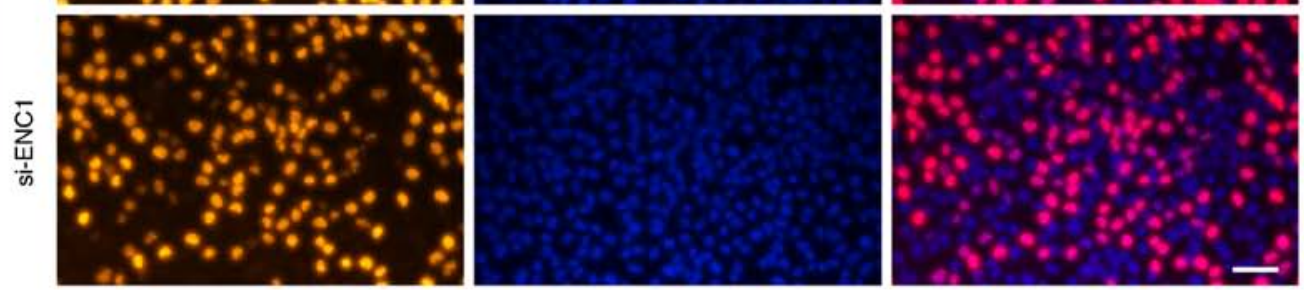

E
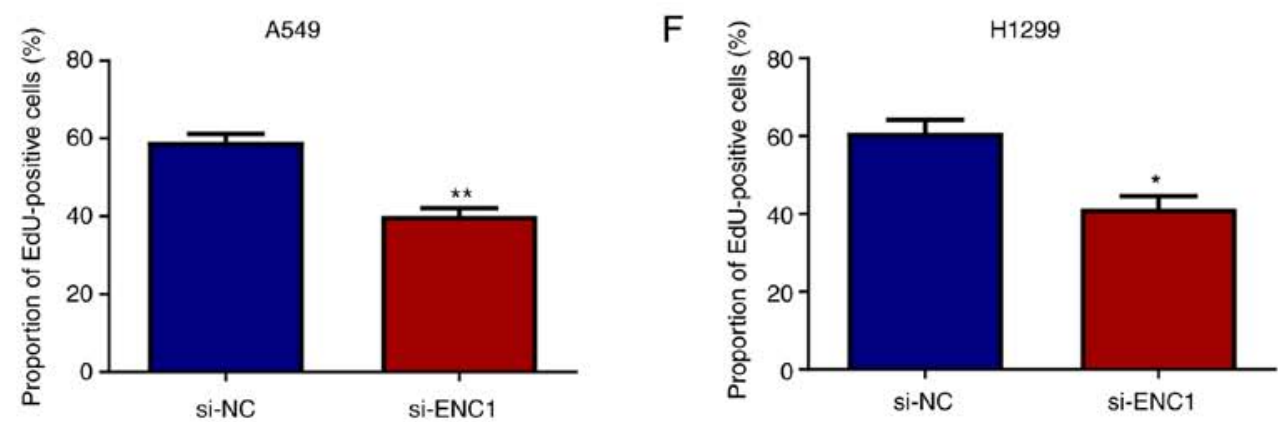

Figure 4. Downregulation of ENC1 inhibits the proliferation of NSCLC cells. (A and B) Effects of ENC1 deficiency on the proliferation were investigated in A549 and $\mathrm{H} 1299$ by CCK-8 assay. (C-F) The effects of ENC1 deficiency on the proliferation were investigated in A549 and H1299 using an EdU detection kit. ${ }^{*} \mathrm{P}<0.05,{ }^{* * *} \mathrm{P}<0.01$ vs. si-NC group. ENC1, ectodermal-neural cortex 1 ; NSCLC, non-small cell lung cancer.

and invasion of A549 and H1299 cells compared with the control group. These results suggest that ENC1 plays a significant role in proliferation, migration and invasion of NSCLC cells.

\section{Discussion}

With over a million deaths annually worldwide, lung cancer is the leading cause of cancer-related mortality $(17,18)$. NSCLC 

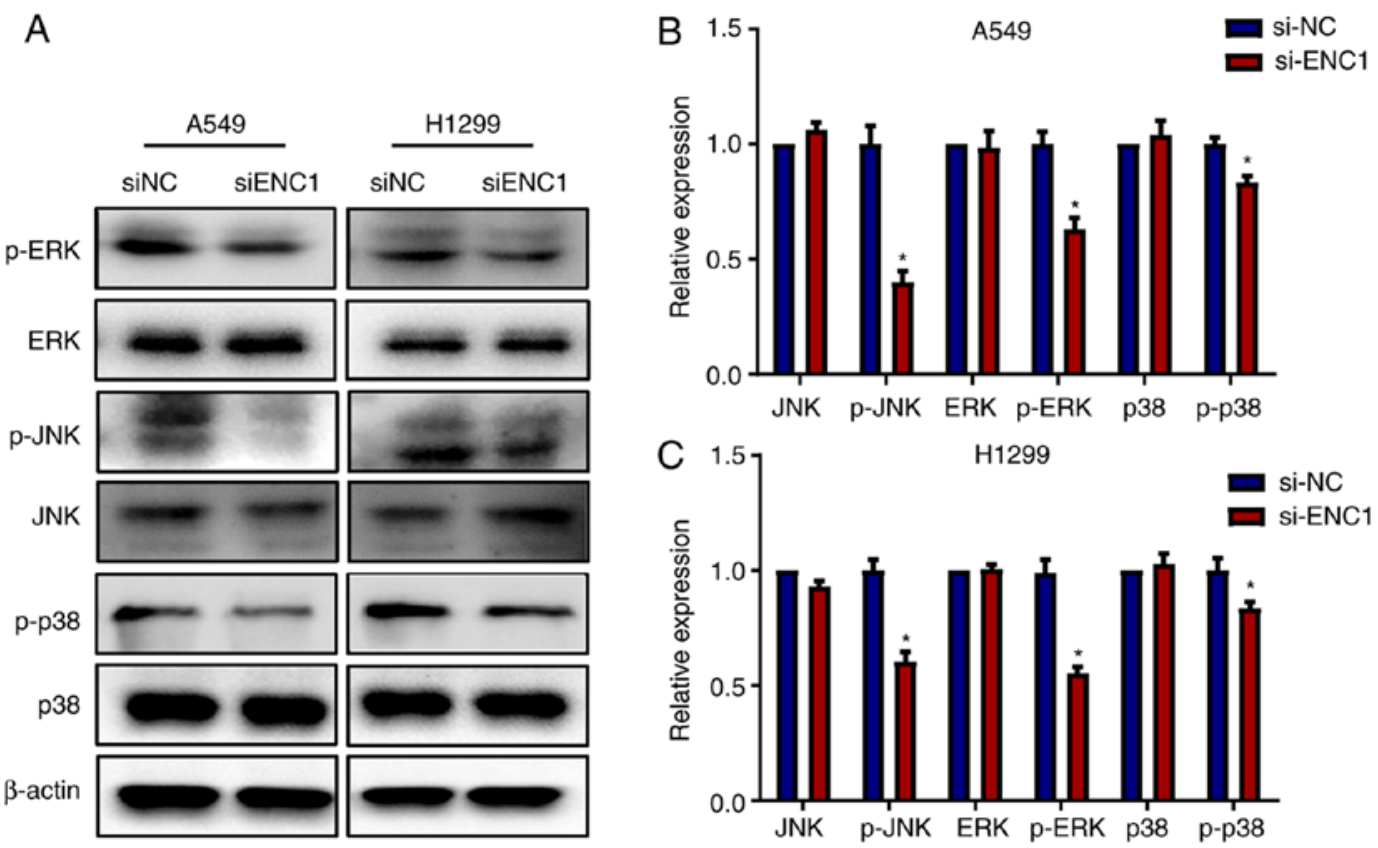

Figure 5. Downregulation of ENC1 affects the JNK and ERK pathways. (A-C) Effects of ENC1 deficiency on the expression of p-ERK, ERK, p-JNK, JNK p-p38 and p38 were quantified in A549 and $\mathrm{H} 1299$ cells by western blot analysis. ${ }^{*} \mathrm{P}<0.05$ vs. si-NC group. ENC1, ectodermal-neural cortex 1.
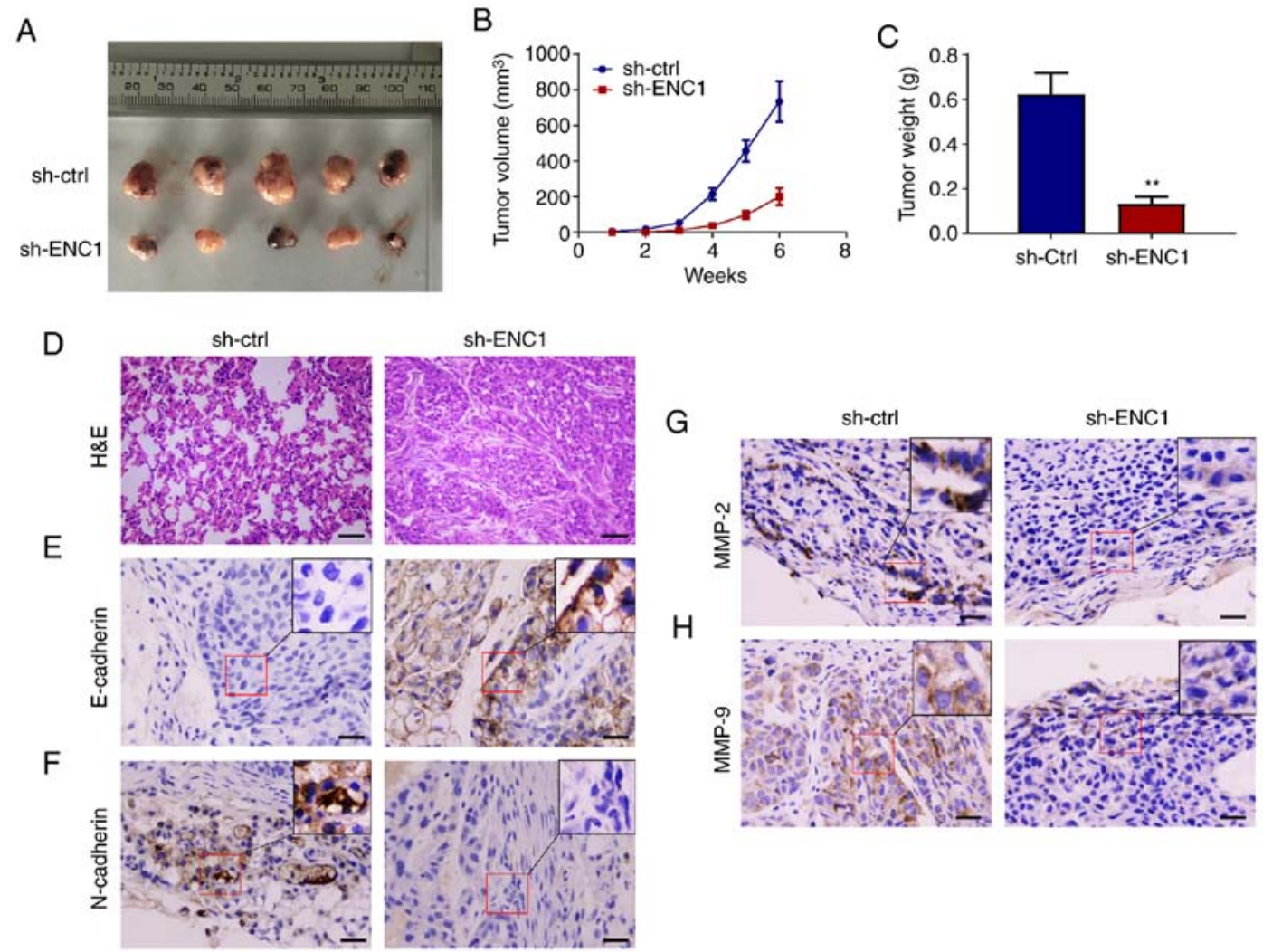

Figure 6. Downregulation of ENC1 inhibits the growth of NSCLC in a mouse model. (A) Image of murine tissues from the 2 groups. (B) Tumor volume in the sh-ctrl and sh-ENC1 groups. (C) Tumor weight in the sh-ctrl and sh-ENC1 groups. (D) Tissue pathological changes in the sh-ctrl and sh-ENC1 groups, determined by H\&E staining. (E-H) The expression levels of N-cadherin, E-cadherin, MMP2 and MMP9 were investigated and compared between the sh-ctrl and sh-ENC1 groups by immunohistochemistry. Scale bar, $50 \mu \mathrm{m} ;{ }^{* * *} \mathrm{P}<0.01$, vs. sh-ctrl. ENC1, ectodermal-neural cortex 1; NSCLC, non-small cell lung cancer.

accounts for approximately $85 \%$ of all lung cancers (19). Despite tremendous advances being made in the treatment using a combination of surgical techniques, chemotherapy and radiotherapy, NSCLC is still associated with a dismal prognosis due to its resistance to therapy and its local recurrence. Consequently, patients with NSCLC have a median survival of $<1$ year and a 2 -year survival rate of $<20 \%$. The lack of effective means for the early diagnosis of NSCLC 


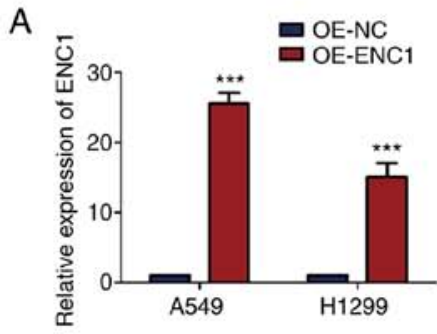

B

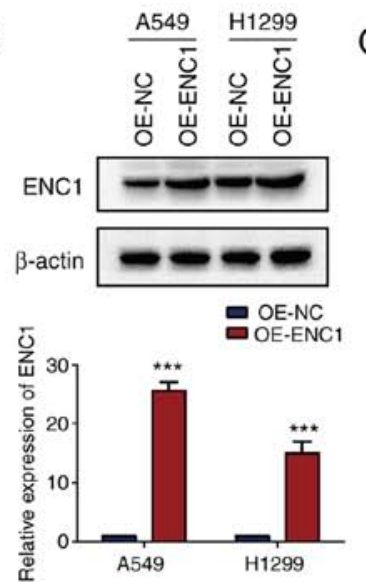

D
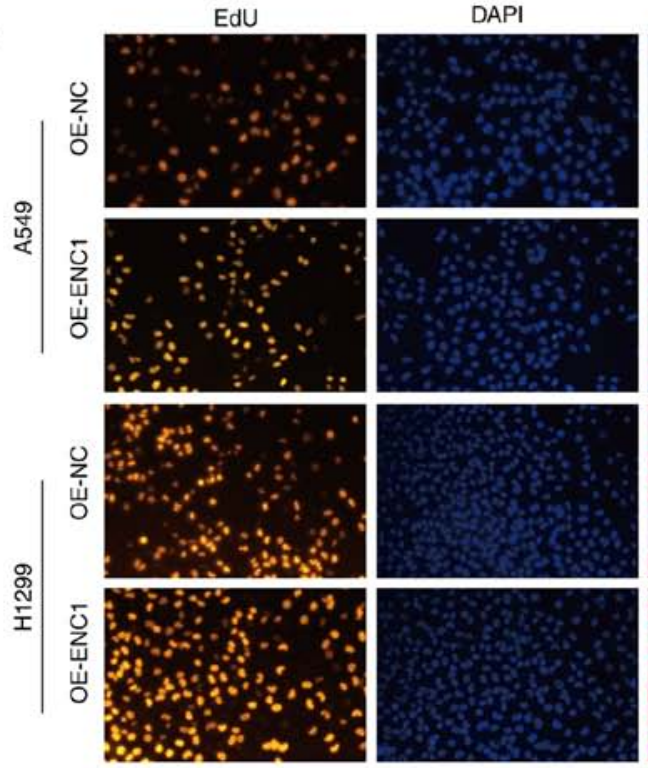

Migration

E
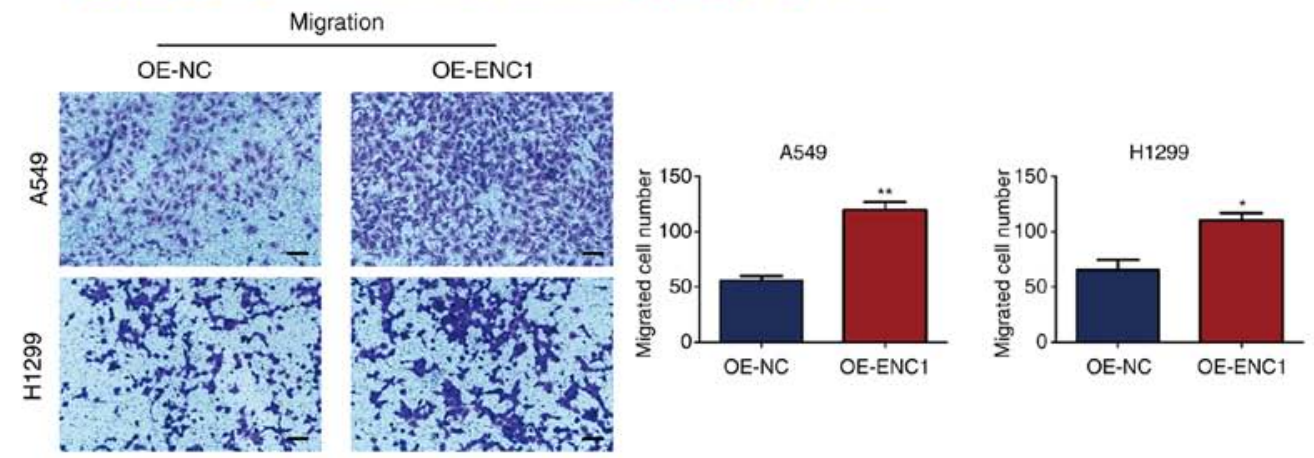
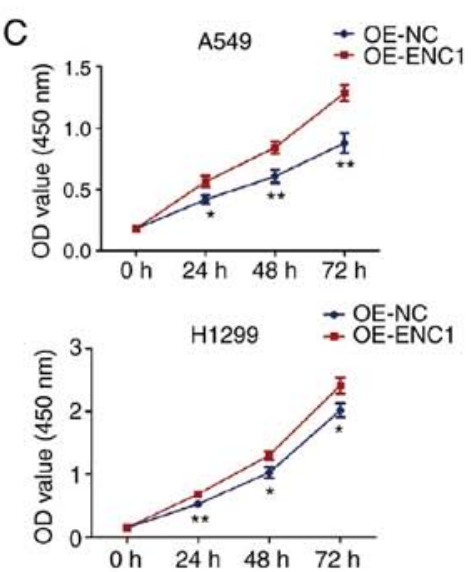
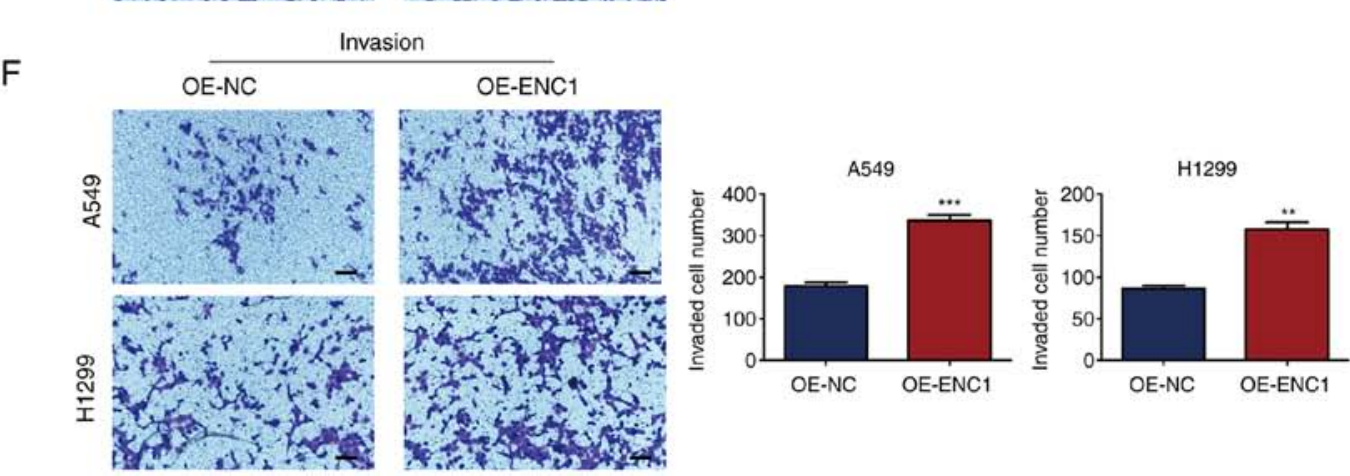

Figure 7. Upregulation of ENC1 enhances the proliferation, migration and invasion of NSCLC cells. (A) The overexpression efficiency of ENC1 was quantified in A549 and H1299 by RT-qPCR. (B) The overexpression efficiency of ENC1 was quantified in A549 and H1299 by western blot analysis. (C) Effects of ENC1 overexpression on proliferation were investigated in A549 and H1299 cells by CCK-8 assay. (D) The effects of ENC1 overexpression on the proliferation were investigated in A549 and H1299 cells using an EdU detection kit. (E and F) The effects of ENC1 overexpression on cell migration and invasion were investigated by Transwell assay. ${ }^{*} \mathrm{P}<0.05,{ }^{* *} \mathrm{P}<0.01,{ }^{* * * *} \mathrm{P}<0.001$ vs. OE-NC group. ENC1, ectodermal-neural cortex 1 ; NSCLC, non-small cell lung cancer. 
is the cause for the high mortality rate associated with this disease $(20,21)$. It is thus critical to identify accurate and sensitive biomarkers for NSCLC in order to increase the survival rate of patients and to lower the costs of treatment.

ENC1 encodes an actin-binding protein and plays an important role in early gastrulation and in the formation of the nervous system. ENC1 is emerging as a novel biomarker due to its conservation, abundance and roles in cancer progression. It is overexpressed in various types of cancer, including ovarian cancer and breast cancer, increasing tumor metastases in patients $(11,12)$. Previous studies have demonstrated controversial associations between ENC1 expression and different types of cancers. ENC1 expression is downregulated in null cell adenomas and oncocytomas (22), but is overexpressed in breast cancer tissue compared with normal breast tissues (12). A high ENC1 expression is associated with a high metastatic ability of breast cancer cells, which was consistent with the finding that ENC1 was associated with the invasiveness of both pituitary null cell adenoma and oncocytoma (12). However, there is a lack of studies demonstrating the association between ENC1 and lung cancer. In the present study, it was demonstrated that the inhibition of ENC1 may be a possible therapeutic target for the treatment of lung cancer. Furthermore, it was found that the downregulation of ENC1 in A549 and H1299 cells by siRNA significantly inhibited their proliferative, migratory and invasive ability, and also affected the expression of proteins MPP2, MMP9, N-cadherin and E-cadherin, which are closely related to the invasion and migration of cancer cells. These results suggested that ENC1 may become another novel diagnostic, metastatic and prognostic biomarker, and even a target for lung cancer in the future.

The MAPK pathway regulates important cellular processes, including gene expression, cell proliferation, cell movement and apoptosis, which play important roles in the progression of NSCLC (23). In the present study, the knockdown of ENC1 expression significantly reduced the phosphorylation levels of 3 MAPK family members, JNK, ERK and p38 in A549 and H1299 cells. Thus, si-ENC1 reduces the proliferation, migration and invasion of lung cancer cells through the MAPK pathway. However, further investigations into the details of ENC1 function are warranted to support these findings. Mouse models will be a valuable resource for identifying therapeutic targets, preclinical screening and the evaluation of different therapies for various types of cancer. It was confirmed that ENC1 regulated the growth and invasion of tumors in nude mice. The results obtained in vivo demonstrated that tumor sizes in nude mice injected with cells transfected with sh-ENC1 were smaller than those of the control group.

In conclusion, the present study demonstrates that ENC1 is significantly overexpressed in lung cancer and has diagnostic and prognostic value. ENC1 may thus be a target for the detection and treatment of NSCLC, as well as other types of cancer.

\section{Acknowledgements}

Not applicable.

\section{Funding}

No funding was received.

\section{Availability of data and materials}

The microarray data has been deposited at the GEO database (GEO-GSE165972). Other raw data supporting the conclusions of the present study will be made available by the authors, without undue reservation, to any qualified researcher.

\section{Authors' contributions}

$\mathrm{CW}$ and $\mathrm{XC}$ conceived and designed the study. CW, XWa and $\mathrm{XWu}$ performed the experiments. CW and $\mathrm{XWa}$ analyzed the data. CW wrote the manuscript. XC edited the manuscript. All authors have read and approved the final manuscript.

\section{Ethics approval and consent to participate}

The present study was approved by the Ethics Committee of Yijishan Hospital. Informed consent was obtained from all patients. The animal research was approved by the Ethics Review Committee of Wannan Medical College.

\section{Patient consent for publication}

Not applicable.

\section{Competing interests}

The authors declare that they have no competing interests.

\section{References}

1. Bray F, Ferlay J, Soerjomataram I, Siegel RL, Torre LA and Jemal A: Global cancer statistics 2018: GLOBOCAN estimates of incidence and mortality worldwide for 36 cancers in 185 countries. CA Cancer J Clin 68: 394-424, 2018.

2. Torre LA, Bray F, Siegel RL, Ferlay J, Lortet-Tieulent J and Jemal A: Global cancer statistics, 2012. CA Cancer J Clin 65: 87-108, 2015.

3. Pfannschmidt J, Muley T, Bülzebruck H, Hoffmann H and Dienemann H: Prognostic assessment after surgical resection for non-small cell lung cancer: Experiences in 2083 patients. Lung Cancer 55: 371-377, 2007.

4. Reungwetwattana T, Weroha SJ and Molina JR: Oncogenic pathways, molecularly targeted therapies, and highlighted clinical trials in non-small-cell lung cancer (NSCLC). Clin Lung Cancer 13: 252-266, 2012.

5. Karuppasamy R, Veerappapillai S, Maiti S, Shin WH and Kihara D: Current progress and future perspectives of polypharmacology: From the view of non-small cell lung cancer. Semin Cancer Biol 68: 84-91, 2021.

6. Hernandez MC, Andres-Barquin PJ, Holt I and Israel MA: Cloning of human ENC-1 and evaluation of its expression and regulation in nervous system tumors. Exp Cell Res 242: 470-477, 1998.

7. Zhao L, Gregoire F and Sul HS: Transient induction of ENC-1, a Kelch-related actin-binding protein, is required for adipocyte differentiation. J Biol Chem 275: 16845-16850, 2000.

8. Lee H, Ahn HH, Lee W, Oh Y, Choi H, Shim SM, Shin J and Jung YK: ENC1 modulates the aggregation and neurotoxicity of mutant huntingtin through p62 under ER stress. Mol Neurobiol 53: 6620-6634, 2016.

9. Kim TA, Ota S, Jiang S, Pasztor LM, White RA and Avraham S: Genomic organization, chromosomal localization and regulation of expression of the neuronal nuclear matrix protein NRP/B in human brain tumors. Gene 255: 105-116, 2000.

10. Fujita M, Furukawa Y, Tsunoda T, Tanaka T, Ogawa M and Nakamura Y: Up-regulation of the ectodermal-neural cortex 1 (ENC1) gene, a downstream target of the beta-catenin/T-cell factor complex, in colorectal carcinomas. Cancer Res 61: 7722-7726, 2001. 
11. Fan S, Wang Y, Sheng N, Xie Y, Lu J, Zhang Z, Shan Q, Wu D, Sun C, Li M, et al: Low expression of ENC1 predicts a favorable prognosis in patients with ovarian cancer. J Cell Biochem 120: 861-871, 2019.

12. Zhou Y,Tang X, Niu L,Liu Y,Wang B and He J: Ectodermal-neural cortex 1 as a novel biomarker predicts poor prognosis and induces metastasis in breast cancer by promoting Wnt/ $\beta$-catenin pathway. J Cell Mol Med 24: 8826-8835, 2020.

13. Livak KJ and Schmittgen TD: Analysis of relative gene expression data using real-time quantitative PCR and the 2(-Delta Delta C(T)) method. Methods 25: 402-408, 2001.

14. Jodele S, Chantrain CF, Blavier L, Lutzko C, Crooks GM, Shimada H, Coussens LM and Declerck YA: The contribution of bone marrow-derived cells to the tumor vasculature in neuroblastoma is matrix metalloproteinase- 9 dependent. Cancer Res 65: 3200-3208, 2005.

15. Mott JD and Werb Z: Regulation of matrix biology by matrix metalloproteinases. Curr Opin Cell Biol 16: 558-564, 2004

16. Leinonen T, Pirinen R, Böhm J, Johansson R, Ropponen K and Kosma VM: Expression of matrix metalloproteinases 7 and 9 in non-small cell lung cancer. Relation to clinicopathological factors, beta-catenin and prognosis. Lung Cancer 51: 313-321, 2006.

17. Li MY, Liu LZ and Dong M: Progress on pivotal role and application of exosome in lung cancer carcinogenesis, diagnosis, therapy and prognosis. Mol Cancer 20: 22, 2021.
18. Han J, Liu Y, Yang S, Wu X, Li H and Wang Q: MEK inhibitors for the treatment of non-small cell lung cancer. J Hematol Oncol 14: 1, 2021.

19. Baade PD, Youlden DR and Krnjacki LJ: International epidemiology of prostate cancer: Geographical distribution and secular trends. Mol Nutr Food Res 53: 171-184, 2009.

20. Reck M, Heigener DF, Mok T, Soria JC and Rabe KF: Management of non-small-cell lung cancer: Recent developments. Lancet 382: 709-719, 2013.

21. Gottesman MM, Lavi O, Hall MD and Gillet JP: Toward a better understanding of the complexity of cancer drug resistance. Annu Rev Pharmacol Toxicol 56: 85-102, 2016.

22. Feng J,Hong L, Wu Y,LiC, Wan H,LiG, Sun Y, Yu S, Chittiboina P, Montgomery B, et al: Identification of a subtype-specific ENC1 gene related to invasiveness in human pituitary null cell adenoma and oncocytomas. J Neurooncol 119: 307-315, 2014.

23. Chang L and Karin M: Mammalian MAP kinase signalling cascades. Nature 410: 37-40, 2001

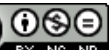

This work is licensed under a Creative Commons Attribution-NonCommercial-NoDerivatives 4.0 International (CC BY-NC-ND 4.0) License. 